\title{
A spectroscopic follow-up for Gaia19bld ${ }^{\star}$
}

\author{
E. Bachelet ${ }^{1} \oplus$, P. Zieliński², M. Gromadzki² ${ }^{2}$ I. Gezer ${ }^{2}$, K. Rybicki² ${ }^{2}$ K. Kruszyńska² ${ }^{2}$ N. Ihanec ${ }^{2}$, Ł. Wyrzykowski $^{2}$, \\ R. A. Street ${ }^{1}$, Y. Tsapras ${ }^{3}$, M. Hundertmark ${ }^{3}$, A. Cassan ${ }^{4}$, D. Harbeck ${ }^{1}$, and M. Rabus ${ }^{1,5}$ \\ ${ }^{1}$ Las Cumbres Observatory, 6740 Cortona Drive, Suite 102,93117 Goleta, CA, USA \\ e-mail: etibachelet@gmail.com \\ 2 Astronomical Observatory, University of Warsaw, Al. Ujazdowskie 4, 00-478 Warszawa, Poland \\ ${ }^{3}$ Zentrum für Astronomie der Universität Heidelberg, Astronomisches Rechen-Institut, Mönchhofstr. 12-14, 69120 Heidelberg, \\ Germany \\ ${ }^{4}$ Institut d'Astrophysique de Paris, Sorbonne Université, CNRS, UMR 7095, 98 bis bd Arago, 75014 Paris, France \\ ${ }^{5}$ Department of Physics, University of California, Santa Barbara, CA 93106-9530, USA
}

Received 28 September 2020 / Accepted 1 July 2021

\begin{abstract}
Context. Due to their scarcity, microlensing events in the Galactic disk are of great interest and high-cadence photometric observations, supplemented by spectroscopic follow-up, are necessary for constraining the physical parameters of the lensing system. In particular, a precise estimate of the source characteristics is required to accurately measure the lens distance and mass.

Aims. We conducted a spectroscopic follow-up of microlensing event Gaia19bld to derive the properties of the microlensing source and, ultimately, to estimate the mass and distance of the lens.

Methods. We obtained low- and high-resolution spectroscopy from multiple sites around the world during the course of the event. The spectral lines and template matching analysis has led to two independent, consistent characterizations of the source.

Results. We found that the source is a red giant located at $\sim 8.5 \mathrm{kpc}$ from the Earth. Combining our results with the photometric analysis has led to a lens mass of $M_{1} \sim 1.1 M_{\odot}$ at a distance of $D_{1} \sim 5.5 \mathrm{kpc}$. We did not find any significant blend light in the spectra (with an upper detection limit of $V \leq 17 \mathrm{mag}$ ), which is in agreement with photometric observations. Therefore, we cannot exclude the possibility that the lens is a main-sequence star. Indeed, we predict in this scenario a lens brightness of $V \sim 20$ mag, a value that would make it much fainter than the detection limit.
\end{abstract}

Key words. gravitational lensing: micro - techniques: spectroscopic - stars: fundamental parameters

\section{Introduction}

The gravitational microlensing method is a powerful way to explore the population of faint objects in the Milky Way. In particular, it has been used over the last $15 \mathrm{yr}$ to discover cold exoplanets towards the Galactic Bulge (89 to date, according to the NASA Exoplanet Archive ${ }^{1}$ ). One of the challenges of this method is to obtain an accurate measurement of the lens mass, $M_{1}$, and distance, $D_{1}$ (Tsapras 2018). Nowadays, about $50 \%$ of the published lens systems masses are dependent on galactic models of the Milky Way (Penny et al. 2016). To estimate the mass of the lens, it is necessary to obtain constraints on at least two mass/distance relations. The first relation can be derived by measuring finite source effects in the lightcurve (Witt \& Mao 1994), parameterized as $\rho$, ultimately leading to the measurement of the size of the angular Einstein ring radius, $\theta_{\mathrm{E}}$ (Yoo et al. 2004). Another route to measuring $\theta_{\mathrm{E}}$ is long-baseline interferometry (Cassan \& Ranc 2016), which up to now, has been achieved for two microlensing events: Kojima-1Lb (Dong et al. 2019) and Gaia19bld (Cassan et al. 2021, C21 thereafter). We can also estimate $\theta_{\mathrm{E}}$ by measuring the movement of the light centroid during the course of the microlensing event, referred to as astrometric microlensing (Dominik \& Sahu 2000). This phenomenon

\footnotetext{
* Data are only available at the CDS via anonymous ftp to cdsarc.u-strasbg.fr $(130.79 .128 .5)$ or via http://cdsarc. u-strasbg.fr/viz-bin/cat/J/A+A/657/A17

1 https://nexsci.caltech.edu/
}

should be systematically achievable for events observed by the Gaia space mission (Gaia Collaboration 2016) with $G \leq 16$ mag and a lens mass $M_{1} \geq 10 M_{\odot}$ (Lu et al. 2016; Rybicki et al. 2018). The measurement of the microlensing parallax, $\pi_{\mathrm{E}}$, provides a second mass mass/distance relation when the source distance $D_{\mathrm{s}}$ is known, since $\pi_{\text {rel }}=\pi_{\mathrm{E}} \theta_{\mathrm{E}}$ and $\pi_{\text {rel }}=1 / D_{1}-1 / D_{\mathrm{s}}$ (Smith et al. 2003). This can be measured via the "annual parallax" (Gould 2004) if the Einstein ring crossing time, $t_{\mathrm{E}}$, is significantly longer than the Earth orbital period or via using the "space parallax", with joint observation from distant observatories, such as Spitzer and ground telescopes (Udalski et al. 2015; Street et al. 2016). Two extra constraints on the lens mass and distance can be obtained with the use of high-resolution imaging to measure the lens flux and lens-source separation several years after the event peak (Beaulieu et al. 2016).

In every case, it is requisite to obtain strong constraints on the source star. In particular, the angular source radius is used to derive the Einstein ring radius since $\theta_{\mathrm{E}}=\theta_{*} / \rho$. For events towards the Galactic Bulge, $\theta_{*}$ is generally derived from the analysis of the color-magnitude diagram (CMD) of the field, which provides an estimate of the reddening, and color-radius relations (Kervella \& Fouqué 2008; Boyajian et al. 2014; Adams et al. 2018). This method, however, cannot be used for sources in the Galactic disk because of the lower stellar density and the higher dispersion of star distances. For such events, spectroscopy is mandatory for constraining the properties and distance of the source. We note that it is often challenging to use the Gaia DR2 
distance estimates (Luri et al. 2018; Bailer-Jones et al. 2018) for microlensing analysis, because of high blending in Galactic disk fields and the nuisance in the astrometric solution due to the astrometric microlensing signal (Rybicki et al. 2018). This can be quantify using the renormalized unit weight error (RUWE) values $^{2}$. It is also common that the microlensing source is missing from the Gaia catalogue due to the high density of stars in microlensing fields.

The use of spectroscopy in microlensing is challenging due to the faintness of the targets and the high stellar density. However, it has been performed on several occasions. The photometric and spectroscopic observations agree at the $\sim 1 \sigma$ level for the source properties of the planetary event MOA-2010-BLG477Lb (Bachelet et al. 2012). Bensby et al. (2013) analyzed the metallicity distribution for a sample of 58 dwarfs and sub-giants sources located in the Bulge using high-resolution spectroscopy, demonstrating a good agreement with the CMD method. With the current facilities, it is also possible to verify microlensing predictions with radial velocity follow-up of close $(\leq 1 \mathrm{kpc})$ binary lenses. The first test done by Boisse et al. (2015) for OGLE-2017-BLG-0417 L was unsuccessful and no modulation was detected. Their null result was supported by high-resolution imaging and near-infrared spectroscopy (Santerne et al. 2016). Ultimately, Bachelet et al. (2018) resolved this puzzle by reanalyzing the microlensing lightcurves, finding a more distant lens than originally estimated. A second test of radial velocity follow-up confirmed the original binary model from a microlensing observation of the event OGLE-2009-BLG-020 L (Skowron et al. 2011; Yee et al. 2016). Spectroscopic observations obtained during the magnification of Kojima-1Lb (Fukui et al. 2019) allowed for an accurate estimation of the lens system, composed of a Super-Earth orbiting a K/M dwarf at $~ 500 \mathrm{pc}$ in the direction of the Taurus constellation. This result was confirmed by the first resolution of microlensing images ever made, using the GRAVITY instrument installed on the Very Large Telescope Interferometer (VLTI) (Dong et al. 2019). Similarly, Wyrzykowski et al. (2020) used spectroscopic observations to constrain the physical properties and distance of the giant source star in the event Gaia16aye.

The alert for the microlensing event Gaia19bld was relayed on April 18, 2019 by the Gaia Science Alerts ${ }^{3}$ (Wyrzykowski \& Hodgkin 2012; Hodgkin et al. 2013) and later recognized as a potential high-magnification event of a bright target located in the Galactic disk, in the direction of the Sagittarius Arm $\left(I \sim 13.5 \mathrm{mag}, l=301.52358^{\circ}, b=-3.27762^{\circ}\right.$ ) (Rybicki et al. 2019). Microlensing events located towards the Galactic disk are rarer than those taking place towards the Galactic Bulge (Han 2008; Sajadian \& Poleski 2019; Mróz et al. 2020), but the former offer several advantages that aid in their characterization. Indeed, they generally have larger $\theta_{\mathrm{E}}$ and $t_{\mathrm{E}}$, which make the previously discussed measurements somewhat simpler.

In addition to the unusual location in the sky, Gaia19bld presented several characteristics that make unique microlensing measurements possible, explored in three follow-up studies. The dense photometric coverage obtained from various observatories allowed for the measurement of $\theta_{\mathrm{E}}$ and $\pi_{\mathrm{E}}$, as described in Rybicki et al. (2022) (hereafter R21). Because the event peak magnitude was so bright, with $H \lesssim 10 \mathrm{mag}$, it has been posssible, for the first time, to observe the microlensing images moving

\footnotetext{
2 https://gea.esac.esa.int/archive/documentation/GDR2/ Gaia_archive/chap_datamodel/sec_dm_main_tables/ssec_ dm_ruwe.html

3 http://gsaweb.ast.cam.ac.uk/alerts/alert/Gaia19bld
}

around the Einstein ring via interferometric measurements. The analysis of the VLT/PIONIER data leading to a direct measure of $\theta_{\mathrm{E}}$ is detailed in Cassan et al. (2021). The extreme brightening of the source has also made intensive spectroscopic follow-up studies possible, and this ultimately allowed for the precise estimation of the source properties described in this paper. The description of the data sets is presented in Sect. 2. Our analysis and results are detailed in Sect. 3. We report our conclusions in Sect. 4.

\section{Observations and data reduction}

\subsection{LCO low-resolution spectra: FLOYDS}

As the event displayed an increased brightness, spectroscopic follow-up observations were immediately scheduled. Lowresolution spectra $(R \sim 500)$ were obtained using the FLOYDS spectrograph, which is mounted on the Las Cumbres Observatory (LCO) 2-m telescope at Siding Spring Observatory (Brown et al. 2013). The spectral range of FLOYDS is approximately 3200-10000 $\AA$, and a slit width of $1.2^{\prime \prime}$ was used here. Because FLOYDS spectra suffer from fringing in the reddest parts, the spectral range was limited to $\lambda \leq 7500 \AA$ in this work. Our goal was to obtain a time series of spectra to extract the spectrum of the source and the blend. We obtained three spectra around the peak of the event: on July 15, 2019 at a magnification of 55.3; on July 19, 2019 at a magnification of 36.6; and on August 1, 2019 at a magnification of 6.94 . We acquired a fourth and final spectrum on February 28, 2020 at a magnification of 1.04 (proposal ID: LCO2019B-014). All spectra were reduced using the LCO FLOYDS pipeline ${ }^{4}$ and they are presented in Fig. 1.

While the primary purpose of the FLOYDS low-resolution spectrum is to discard possible contaminants, it can confirm the spectral type of the source if the extinction is moderate towards the event (Fukui et al. 2019). Unfortunately, in the present analysis, the spectra are affected by two major flaws. Around peak magnification, when the first two spectra were obtained, the Moon was bright (at phases of 98 and 93\%, respectively). This drastically affected the acquisition and reduction of the observations. As the event passed the peak, the last two spectra were acquired while the event was dimmer, leading to suboptimal guiding. The effects of these two phenomena can be seen in Fig. 2. Since the field suffers from severe extinction, the measured signal in the given spectral range has a moderate signal-to-noise ratio $(S / N \leq 15)$. Therefore, we rejected these spectra from the template matching modeling described in Sect. 3.2, but we note that they are in good agreement with the derived source spectrum, as can be seen in Fig. 1.

\subsection{LCO high-resolution spectra: NRES}

Gaia19bld became bright enough $(V \leq 12 \mathrm{mag})$ that it was possible to trigger high-resolution spectroscopy with the LCO telescopes; LCO recently deployed the Network of Robotic Echelle Spectrographs (NRES) (Siverd et al. 2018), composed of four nearly identical optical high-resolution $(R \sim 53000)$ spectrographs, evenly distributed in both hemispheres. The spectrographs are fed by two sky fibres with $2.9^{\prime \prime}$ width on sky and one simultaneous ThAr calibration fibre. The two sky fibres are attached to two 1-m telescopes at a given site, but only one telescope is selected by the scheduler for a given observation.

4 https://github.com/LCOGT/floyds_pipeline 


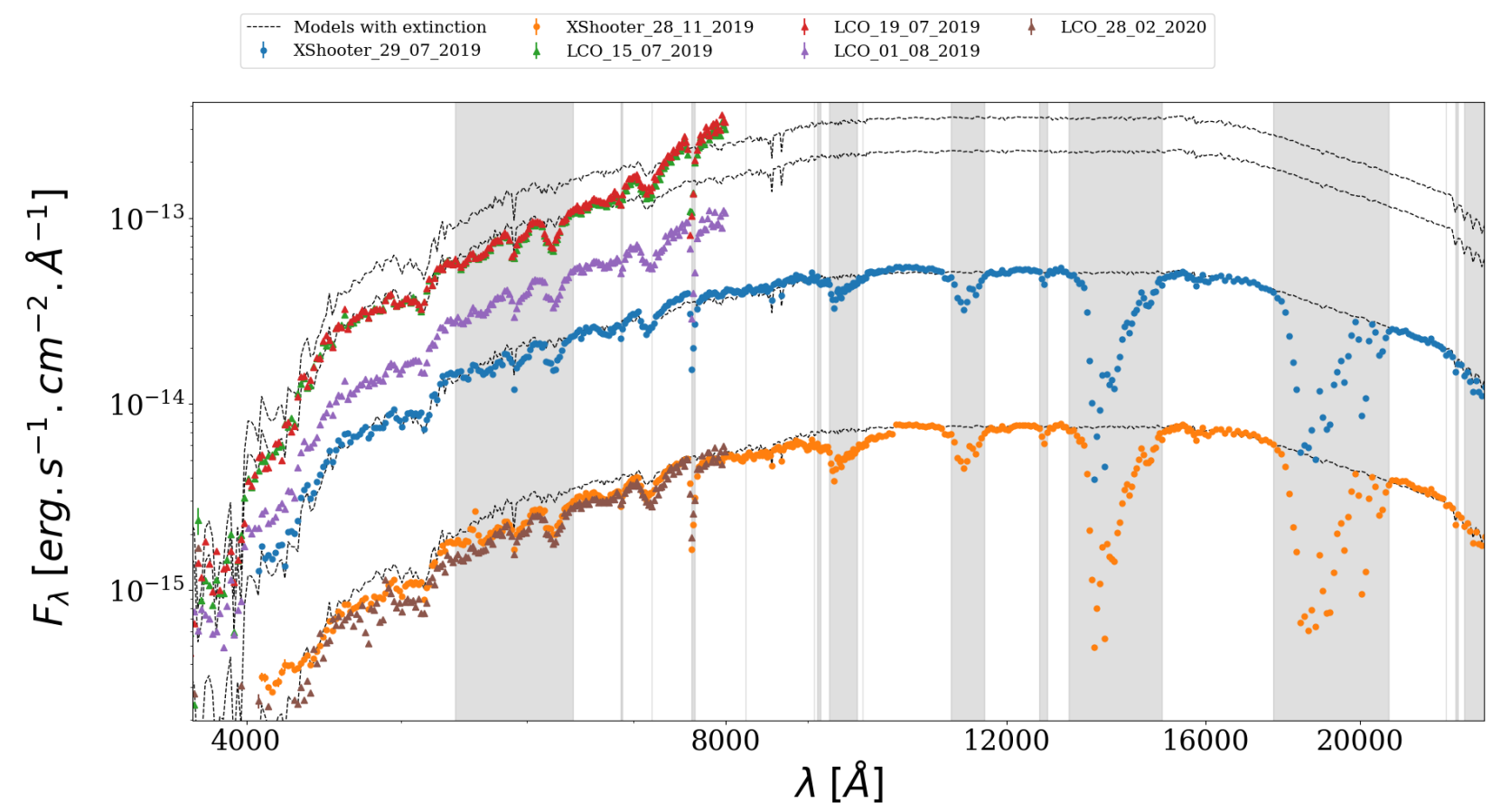

Fig. 1. Four LCO FLOYDS and two X-shooter spectra clearly indicating the magnification of the source. Dash lines indicate the best fit model (model A, displayed for four different magnification levels) from the template-matching analysis, while the grey regions correspond to telluric lines, with absorption $\geq 2 \%$. The LCO FLOYDS spectra confirm the spectral type but were not used for the modeling. The observed spectra have been scaled to the observed magnitude at the time of acquisition (i.e., they represent the spectra as seen at the top of the atmosphere).
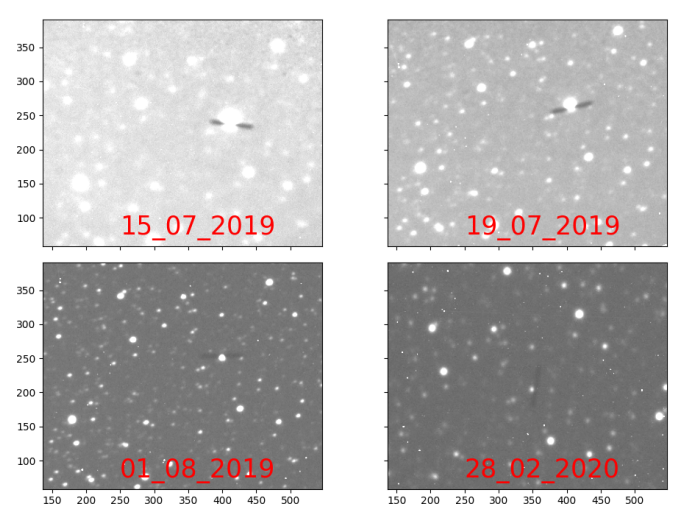

Fig. 2. Examples of guiding frames centered around Gaia19bld for each LCO FLOYDS spectra. The magnification of the source is visible. Top: images displayed with identical scaling, so that the brighter sky background is evident in the top two images. Bottom: decreasing target brightness resulting in sub-optimal robotic guiding.

The NRES observations were done on two occasions: on July 15, 2019 at a magnification of 55.34 and on July 19, 2019 at a magnification of 36.13 (proposal ID: LCO2019B-014). In order to extract the wavelength calibrated spectra, we adapted the CERES pipeline (Brahm et al. 2017) to reduce the NRES spectra. The pipeline starts with a bias and dark calibration of the raw images. Flat-field images are used to create the traces and the flux for each order is extracted using optimal extraction methods, as described in Horne (1986). Finally, each order is wavelength-calibrated using the ThAr calibration fibre.

\subsection{VLT/X-shooter spectra}

In addition, we used the X-shooter instrument (Vernet et al. 2011) mounted on the ESO Very Large Telescope (VLT) which is a multi-wavelength, medium-resolution spectrograph consisted of three spectroscopic arms allowing for simultaneous observations at three wavelength ranges: UVB (300-559.5 nm), VIS (559.5-1024 nm), and NIR (1024-2480 nm). The resolution in each range is different due to an independent cross dispersed elements with own detector's shutters and slit masks. For UVB, VIS, and NIR ranges we were able to obtain $R \sim 5400$ at slit width $1.0^{\prime \prime}, R \sim 11400$ at slit width $0.7^{\prime \prime}$, and $R \sim 8100$ at slit width $0.6^{\prime \prime}$, respectively. The X-shooter spectrograph was used two times: on July 29, 2019 at a magnification of 8.14 (i.e., close to the peak of the microlensing event) and an airmass of 1.47; and on November 28, 2019 at a magnification of 1.21 (i.e., close to the brightness baseline, ESO DDT proposal ID: 2103.D5046) and an airmass of 2.15. The exposure times were 173, 207, and $2 \times 125 \mathrm{~s}$ in the UVB, VIS, and NIR arms, respectively. We reduced the spectra with the dedicated EsoReflex ${ }^{5}$ pipeline (v. 2.9.1). For the calibration of UVB, VIS wavelengths, ThAr lamp was used, while for NIR - a set of $\mathrm{Ar}, \mathrm{Hg}, \mathrm{Ne}$ and Xe lamps. The calibrated X-shooter spectra are presented in Fig. 1.

\section{Properties of the source and the lens}

\subsection{Absorption line analysis}

At first, the spectroscopic analysis of absorption lines for Gaia19bld is performed on two high-quality $(S / N=22$ and 221) $\mathrm{X}$-shooter VIS as well as two NRES spectra. The resolution obtained for all of these data allows for stellar parameters determination thanks to the $i$ Spec $^{6}$ framework for spectral analysis, which integrates several well-known radiative transfer codes (Blanco-Cuaresma et al. 2014; Blanco-Cuaresma 2019).

\footnotetext{
5 https://wwW.eso.org/sci/software/esoreflex/ 6 https://www.blancocuaresma.com/s/iSpec
} 
Table 1. Summary of the derived parameters for the source of Gaia19bld event.

\begin{tabular}{lcccc}
\hline \hline Parameter & Line fitting & \multicolumn{4}{c}{ Template matching } \\
& & A & B & C \\
\hline$T_{\text {eff }}[\mathrm{K}]$ & $4159 \pm 139$ & $4097_{-29}^{+32}$ & $4052_{-28}^{+27}$ & $4091_{-31}^{+31}$ \\
$\log g$ & $1.89 \pm 0.42$ & $1.48_{-0.15}^{+0.16}$ & $1.27_{-0.17}^{+0.15}$ & $1.49_{-0.16}^{+0.15}$ \\
{$[\mathrm{M} / \mathrm{H}][\mathrm{dex}]$} & $0.42 \pm 0.20$ & $0.295_{-0.062}^{+0.053}$ & $0.219_{-0.054}^{+0.050}$ & $0.289_{-0.062}^{+0.055}$ \\
$v_{\mathrm{t}}\left[\mathrm{km} \mathrm{s}^{-1}\right]$ & $2.04 \pm 0.58$ & - & - & - \\
$A_{\mathrm{v}}[\mathrm{mag}]$ & - & $2.322_{-0.072}^{+0.075}$ & $2.153_{-0.070}^{+0.064}$ & $2.214_{-0.076}^{+0.074}$ \\
$R_{\mathrm{v}}$ & - & $3.40_{-0.10}^{+0.11}$ & $3.13_{-0.069}^{+0.072}$ & $3.117_{-0.085}^{+0.089}$ \\
$\theta_{*}[\mu \mathrm{as}]$ & - & $24.16_{-0.40}^{+0.39}$ & $23.20_{-0.38}^{+0.38}$ & $23.11_{-0.55}^{+0.53}$ \\
$k_{1}$ & - & $117.0_{-6.3}^{+7.0}$ & $103.0_{-4.0}^{+4.4}$ & $101.5_{-3.8}^{+4.3}$ \\
$k_{2}$ & - & $68.6_{-3.8}^{+4.0}$ & $48.1_{-1.9}^{+2.0}$ & - \\
$\mathrm{L}$ & - & 21430 & 21582 & 10595 \\
\hline$\theta_{\mathrm{E}}[\mu \mathrm{as}]$ & - & $754_{-13}^{+13}$ & $724_{-12}^{+12}$ & $721_{-18}^{+17}$ \\
$M_{1}\left[M_{\odot}\right]$ & - & $1.126_{-0.026}^{+0.027}$ & $1.081_{-0.025}^{+0.026}$ & $1.076_{-0.031}^{+0.031}$ \\
$D_{\mathrm{s}}[\mathrm{kpc}]$ & - & $8.4_{-1.8}^{+1.3}$ & $9.3_{-1.9}^{+1.5}$ & $8.7_{-1.9}^{+1.4}$ \\
$D_{\mathrm{l}}[\mathrm{kpc}]$ & - & $5.50_{-0.82}^{+0.56}$ & $5.97_{-0.84}^{+0.59}$ & $5.74_{-0.86}^{+0.58}$ \\
\hline
\end{tabular}

Notes. Averaged solutions of line fitting are presented. The three minima $\mathrm{A}, \mathrm{B}$, and $\mathrm{C}$ from the template matching method discussed in the text are presented. The physical parameters $\theta_{\mathrm{E}}, M_{1}, D_{\mathrm{s}}$, and $D_{\mathrm{l}}$ have been estimated using the $\rho$ and $\pi_{E}$ parameters from the light curve modeling presented in R21.

In our case, to determine atmospheric parameters (i.e., effective temperature $T_{\text {eff }}$, surface gravity $\log g$, metallicity $[\mathrm{M} / \mathrm{H}]$, microturbulence velocity $v_{\mathrm{t}}$ ), the $\mathrm{SPECTRUM}^{7}$ code is used.

In order to synthesize theoretical spectra and fit them to observational data, we select prominent atomic lines $(\mathrm{H} \alpha, \mathrm{H} \beta$, $\mathrm{Ca}, \mathrm{Mg}, \mathrm{Fe}$ ), a well-known grid of MARCS atmospheric models (Gustafsson et al. 2008), and solar abundances taken from Grevesse et al. (2007). Due to the fact that the vast majority of atomic lines identified in X-shooter spectra for which we do have precise laboratory data (exact wavelengths, excitation potentials, oscillator strengths values, etc.) are visible in the VIS part, we decided to focus only on this region. The best-matching synthetic spectra are fitted for parameters presented in Table 1. Figure 3 shows X-shooter spectra with fitted synthetic ones around the CaIII triplet region, while Fig. 4 shows the NRES and synthetic spectra around 6480-6520 $\mathrm{A}$ Fe lines. For all spectra, the results are the same within the uncertainties. No absorption lines from a potential second component are visible in the X-shooter nor the NRES data.

Moreover, Jennings \& Levesque (2016) recommend the use of the $\mathrm{H} \alpha$ equivalent width $\mathrm{W}(\mathrm{H} \alpha)$ as a potential class diagnostic for red giants because this parameter is correlated with the overpopulation of the metastable $2 \mathrm{~s}$ level of hydrogen in non-LTE conditions. We computed $\mathrm{W}(\mathrm{H} \alpha)$ for the NRES and $\mathrm{X}$-shooter spectra of Gaia19bld and compared them with values found in Jennings \& Levesque (2016). The weighted average is $\mathrm{W}(\mathrm{H} \alpha)=1.13 \pm 0.08 \AA$ and given $T_{\text {eff }} \sim 4100 \mathrm{~K}$, the source star radius is about $40 R_{\odot}$.

\footnotetext{
7 https://www .appstate.edu/ grayro/spectrum/spectrum. html
}
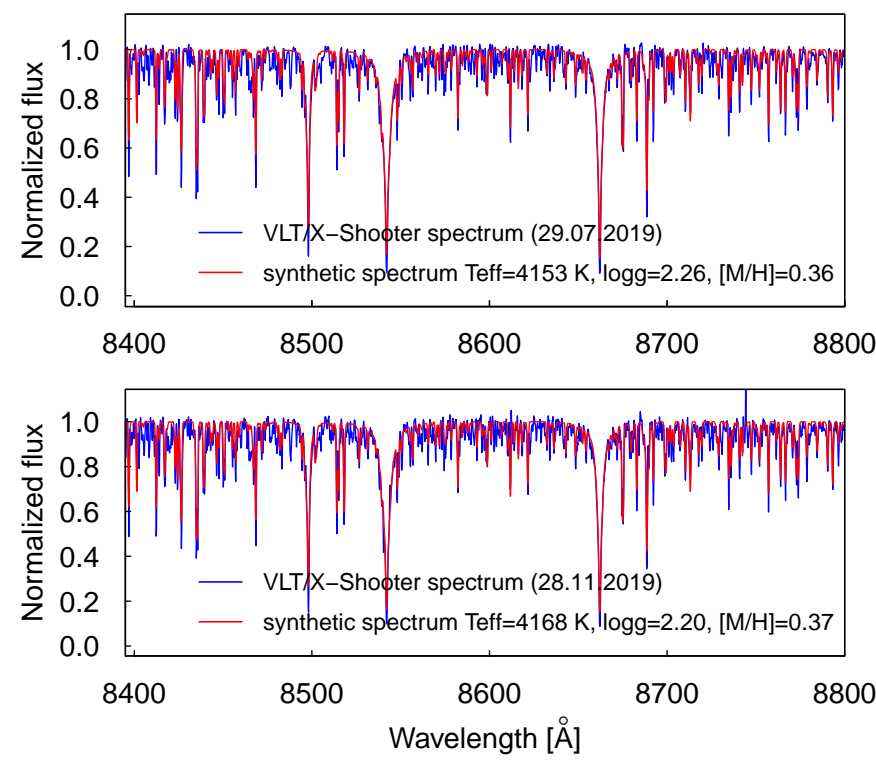

Fig. 3. Normalized X-shooter spectra (blue) obtained in two epochs and synthetic spectra (red) best fits for specific parameters. The Ca II triplet region is visible.
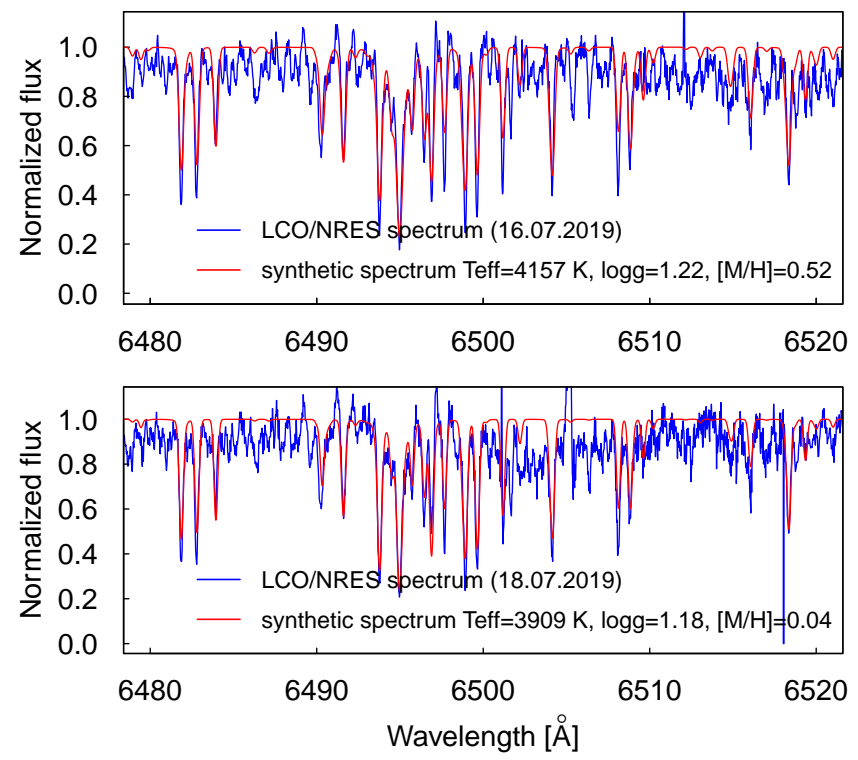

Fig. 4. Normalized NRES spectra (blue) obtained in two epochs and synthetic spectra (red) best fits for specific parameters. The Fe lines region $(6480-6520 \AA)$ is visible.

\subsection{Template matching}

A second way to analyze the spectra at hand is to model the data with spectroscopic templates across the full wavelength range. This approach is complementary to the analysis presented in the previous sections. It allows us to derive an estimation of the angular radius of the source and the extinction along the line of sight. The first step is to re-arrange the data to a common wavelength basis, defined by the one used in the template model. This also considerably increases modeling speed, but introduces correlated noise in the data (Carnall 2017). Then, it is also necessary to calibrate the spectra to match the predicted brightness of the event in different photometric bands. We used the magnification at the time of observation, $A(t)$, and the $I$ and Gaia-G bands to estimate the parameter, $R_{i}$, for each spectrum in our model. This 
is mandatory to derive an accurate measurement of the angular source size since $F_{\lambda} \propto \theta_{*}^{2}$. We therefore included a flux rescaling factor in the spectrum model, used a Gaussian prior, and added a contribution to the likelihood for each spectrum:

$\chi_{b}^{2}=\sum_{b} \frac{\left(y_{b}-m_{b}\right)^{2}}{\sigma_{b}^{2}}$,

where $y_{b}$ and $\sigma_{b}$ are the observed fluxes and errors in the band $b$ at the time of the spectrum acquisition. We assumed a conservative $\sigma=0.1 \mathrm{mag}$ for all photometric measurements.

To model the spectra, we used the pysynphot software (STScI Development Team 2013) for generating the spectral templates from Kurucz (1993). This allowed us to estimate the effective temperature, $T_{\text {eff }}$, the surface gravity, $\log g$, and the metallicity, $[\mathrm{Fe} / \mathrm{H}]$, of the star. To match the model to the data, the angular source radius $\theta_{*}$ is also required and used to scale the flux. Finally, we used the relations from Cardelli et al. (1989) to estimate the extinction towards the source, parameterized by $A_{\mathrm{V}}$ and $R_{\mathrm{V}}$. The microlensing model is defined as:

$f_{\lambda}(t)=f_{\mathrm{s}, \lambda} A(t)+f_{\mathrm{b}, \lambda}$,

where $f_{\mathrm{s}, \lambda}$ and $f_{\mathrm{b}, \lambda}$ are the source and blend fluxes at wavelength $\lambda$. We used the magnification from the photometric models detailed in R21. We consider the errors of the spectra to potentially be underestimated and add to the model the parameter $k$ for each spectrum:

$\sigma_{\lambda}^{\prime}=\sqrt{k^{2} \sigma_{\lambda}^{2}}$

with $\sigma_{\lambda}^{\prime}$ and $\sigma_{\lambda}$ the new and original errors at the wavelength $\lambda$. The terms k take account of the flux calibration errors and can be expected to be on the order of $\sim \frac{\sigma}{\sqrt{N}} S / N$, where the $\mathrm{S} / \mathrm{N}$ is the mean signal-to-noise ratio of the spectra. The two X-shooter spectra have $S / N \sim 1700$ and $S / N \sim 650$ (after binning) and thus we should expect $k_{1} \sim 120$ and $k_{2} \sim 40$, respectively. Ultimately, we model the data by maximizing the likelihood $L$ :

$L=-0.5 \sum_{\lambda}\left(\frac{\left(y_{\lambda}-m_{\lambda}\right)^{2}}{\sigma_{\lambda}^{\prime 2}}+\chi_{b}^{2}+\ln \left(2 \pi \sigma_{\lambda}^{\prime}\right)\right)$.

To explore the posterior distribution, we used the emcee package (Foreman-Mackey et al. 2013). We rejected points with atmospheric extinction higher than $2 \%$ and used the telluric lines defined in Moehler et al. (2014). We identify a single dominant minima, noted $\mathrm{A}$ in Table 1 and visible in the Fig. 1. It can be seen in Fig. 1 that the second X-shooter spectrum present a slight discontinuity starting around $10000 \AA$. A close look at the data reveals a small offset between the calibration of the VIS and NIR arms, which we found to be $\epsilon \sim 0.87$ (a multiplicative offset). The cause of this offset can be due to the observation at low airmass (2.15), where the hardware and software corrections can underperform ${ }^{8}$. After the correction of this offset, we ran a second round of modeling that converges to the solution B. Finally, we found an extra solution, noted C, by entirely removing the second X-shooter spectrum. All solutions indicate that the source is a red giant, with slightly different spectral types and extinction properties for each individual solution. The absorption, $A_{\mathrm{V}}$, derived in model $\mathrm{A}, \mathrm{B}$, and $\mathrm{C}$ are in

8 https://www.eso.org/sci/facilities/paranal/ instruments/xshooter/doc/VLT-MAN-ESO-14650-4942_ P104v1.pdf agreement with the independent estimations, that is, $2.01 \pm 0.03$ and $2.34 \pm 0.04$ from Schlafly \& Finkbeiner (2011) and Schlegel et al. (1998) (both assuming $R_{\mathrm{V}}=3.1^{9}$ ), and $1.88 \pm 0.35$ from Anders et al. (2019). To compare these results with the photometric measurements, we computed the magnitude of the source in several bands for all models. As summarized in Table 2, the flux at the event location is almost solely due to the source. Using the zero-point correction from Blanton \& Roweis (2007) and Weiler (2018), we found the source magnitude to be $G=14.8 \pm 0.2 \mathrm{mag}$, $V=15.7 \pm 0.2 \mathrm{mag}$, and $I=13.4 \pm 0.2 \mathrm{mag}$. This is in excellent agreement with the measurements of R21 with $G=14.8 \mathrm{mag}$, $V=15.9 \mathrm{mag}$, and $I=13.5 \mathrm{mag}$, and it is in good agreement with archival data ${ }^{10}$. All models predict very similar magnitudes in all bands and are in agreement at the $1 \sigma$ level. We note, however, that models $\mathrm{B}$ and $\mathrm{C}$ are in better agreement with the archival near-infrared data from Cutri et al. (2003).

\subsection{Einstein ring radius, source distance, and lens properties}

The photometric analysis presented in R21 was converged to $\rho=0.03198 \pm 0.00016$ and $\pi_{\mathrm{E}}=0.0815 \pm 0.0014$; we used these values to compute the values of $\theta_{\mathrm{E}}$ and $M_{1}$ presented in Table 1. These values are in agreement with the independent measurement $\theta_{\mathrm{E}}=0.765 \pm 0.004$ mas from interferometry (C21), especially for model A.

One of the difficulties of microlensing studies is the estimation of the source distance, $D_{\mathrm{s}}$. This is especially true for fields outside of the Galactic Bulge, where the extinction is not well known. However, the angular radius of the source and fundamental stellar properties derived in previous sections allow us to estimate the source distance with four different methods. Here, we detail the source distance estimate made for model A, however, all the results can be found in Table 1. First, it is clear from the fit parameters that the source in Gaia19bld is a red giant. Therefore, using the empirical relations presented in Sect. 3.1, and from Berger et al. (2018) and Alonso et al. (2000), we can assume the physical radius of the source to be $R_{*}=40 \pm 10 R_{\odot}$. We assumed a relatively low precision on the stellar radius due to the intrinsic scatter in the $T_{\text {eff }}$-radius relation for the red giant population, as well as the interpolation methods used to estimate these radii. We note, however, that using the stellar parameters of the source with the PARSEC isochrones (Bressan et al. 2012) ${ }^{11}$ returns a similar value of $R_{*}=44 \pm 3 R_{\odot}$. This estimation, associated with the posterior distribution of the angular source radius, led to a direct measurement of the source distance: $D s=7.7_{-1.9}^{+1.9}$ $\mathrm{kpc}$. Secondly, using the PARSEC isochrones (Bressan et al. 2012), with a fixed age of $1 \mathrm{Gyr}$, and the derived source parameters, it is possible to estimate the absolute magnitude, $M_{\mathrm{V}} \sim-1.0$ mag and $M_{\mathrm{I}} \sim-2.5 \mathrm{mag}$, of the source. Coupled with the apparent magnitudes of the source, $V=15.85 \mathrm{mag}$ and $I=13.52$ mag, and the absorption law derived from spectral modeling, we obtain two extra source distance estimates: $D s=8.4_{-1.6}^{+0.9} \mathrm{kpc}$ and $D s=8.4_{-1.5}^{+0.8} \mathrm{kpc}$. Finally, we use the expressions from BailerJones et al. (2018) for a fourth independent distance estimate of the source $D s=8.9_{-1.8}^{+2.7} \mathrm{kpc}$. The four resulting distributions for the model A can be seen in Fig. 5. Combining all the distributions gives a distance estimate of $D s=8.4_{-1.8}^{+1.4} \mathrm{kpc}$.

According to Eq. (2), it is possible to extract the source and blend the spectra if at least two measurements were obtained at two different magnifications. We did not find any significant

\footnotetext{
9 https://irsa.ipac.caltech.edu/applications/DUST/

10 https://vizier.u-strasbg.fr/viz-bin/VizieR

11 http://stev.oapd.inaf.it/cgi-bin/cmd
} 
Table 2. Predicted AB magnitudes for the source, the main-sequence lens scenario as well as archival measurements at the event location.

\begin{tabular}{|c|c|c|c|c|c|}
\hline \multirow[t]{2}{*}{ Filter } & \multicolumn{3}{|c|}{ Source } & \multirow[t]{2}{*}{ Main sequence lens } & \multirow[t]{2}{*}{ Archival data } \\
\hline & A & $\mathrm{B}$ & $\mathrm{C}$ & & \\
\hline $\mathrm{G}$ & $14.88(0.08)$ & $14.89(0.07)$ & $14.86(0.06)$ & $19.9(0.5)$ & 14.897(0.003) (Gaia Collaboration 2016,2021$)$ \\
\hline $\mathrm{U}$ & $21.2(0.2)$ & $21.3(0.2)$ & $21.3(0.2)$ & $23.0(0.5)$ & $*$ \\
\hline $\mathrm{B}$ & $17.7(0.1)$ & $17.7(0.1)$ & $17.7(0.1)$ & $21.5(0.6)$ & 17.52 (Girard et al. 2011) \\
\hline $\mathrm{V}$ & $15.8(0.1)$ & $15.8(0.1)$ & $15.8(0.1)$ & $20.3(0.5)$ & 15.99 (Girard et al. 2011) \\
\hline $\mathrm{R}$ & $14.78(0.09)$ & $14.79(0.08)$ & $14.8(0.1)$ & $19.7(0.5)$ & 14.6 (Zacharias et al. 2005) \\
\hline $\mathrm{I}$ & $13.92(0.07)$ & $13.94(0.07)$ & $13.93(0.08)$ & $19.2(0.5)$ & 13.93(0.02) (DENIS Consortium 2005) \\
\hline $\mathrm{u}$ & $21.1(0.2)$ & $21.2(0.2)$ & $21.2(0.2)$ & $23.1(0.6)$ & $*$ \\
\hline $\mathrm{g}$ & $17.0(0.1)$ & $17.1(0.1)$ & $17.1(0.1)$ & $21.1(0.6)$ & $*$ \\
\hline $\mathrm{r}$ & $15.1(0.1)$ & $15.1(0.1)$ & $15.1(0.1)$ & $19.9(0.5)$ & $*$ \\
\hline $\mathrm{i}$ & $14.22(0.08)$ & $14.24(0.07)$ & $14.23(0.09)$ & $19.4(0.5)$ & $*$ \\
\hline $\mathrm{z}$ & $13.50(0.07)$ & $13.54(0.06)$ & $13.52(0.08)$ & $19.0(0.5)$ & $*$ \\
\hline $\mathrm{J}$ & $12.63(0.05)$ & $12.69(0.05)$ & $12.67(0.06)$ & $18.6(0.5)$ & 12.76(0.03) (Cutri et al. 2003) \\
\hline $\mathrm{H}$ & $12.12(0.04)$ & $12.18(0.04)$ & $12.18(0.04)$ & $18.5(0.5)$ & 12.25(0.02) (Cutri et al. 2003) \\
\hline $\mathrm{K}$ & $12.29(0.04)$ & $12.36(0.04)$ & $12.37(0.04)$ & $18.8(0.5)$ & 12.36(0.02)(Cutri et al. 2003) \\
\hline
\end{tabular}

Notes. The number in brackets indicates the standard uncertainties. The archival magnitudes have been corrected to the AB system, using Blanton $\&$ Roweis (2007) and Weiler (2018).

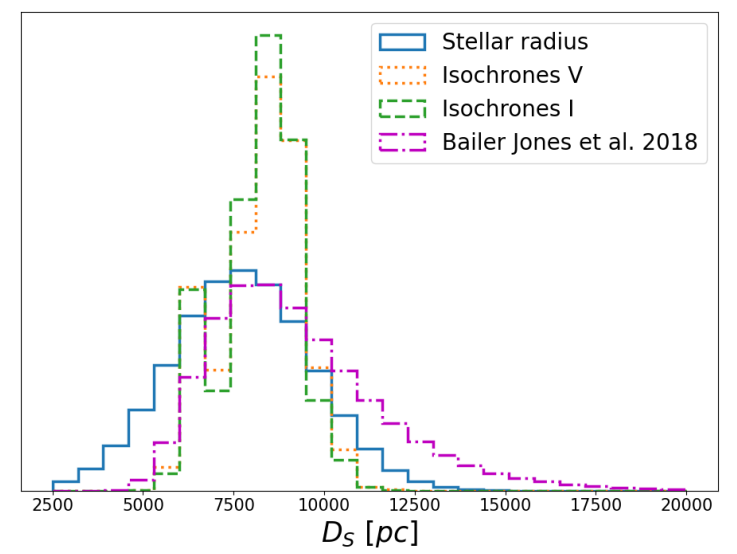

Fig. 5. Distributions of the distance of the source from the various methods detailed in the text (model A).

blend light in this case (after the correction of the offset of the second $\mathrm{X}$-shooter spectrum). It is also possible to estimate the covariance matrix as:

$\mathbf{C}=\mathbf{H}^{-1}$,

where $\mathbf{H}$ is the Hessian matrix which is equal to:

$\mathbf{H}=\mathbf{M}^{\mathbf{T}} \mathbf{M}$,

and

$\mathbf{M}=\left(\begin{array}{ll}\mathbf{A} & \mathbf{B} \\ \mathbf{B} & \mathbf{C}\end{array}\right)$.

Here, $\mathbf{A}, \mathbf{B}$, and $\mathbf{C}$ are diagonal square matrices of dimension $N_{\lambda} \times N_{\lambda}$ (where $N_{\lambda}$ is the total number of observed wavelengths), assuming the $N_{\mathrm{s}}$ spectra have been observed at different magnifications, $A_{\mathrm{s}}$, over the course of the microlensing event. The diagonal of matrices $\mathbf{A}, \mathbf{B}$, and $\mathbf{C}$ can be written as:

$A_{\lambda}=\sum_{i=1}^{N_{\mathrm{s}}} \frac{A_{i}^{2}}{\sigma_{\lambda, i}^{2}} ; B_{\lambda}=\sum_{i=1}^{N_{\mathrm{s}}} \frac{A_{i}}{\sigma_{\lambda, i}^{2}} ; C_{\lambda}=\sum_{i=1}^{N_{\mathrm{s}}} \frac{1}{\sigma_{\lambda, i}^{2}}$.
Using the two X-shooter spectra, we estimate the error on the measured source and blend fluxes to be $\sigma_{f_{\mathrm{s}, \lambda}} \sim 3 \times$ $10^{-16} \mathrm{erg} \mathrm{s}^{-1} \mathrm{~cm}^{-2} \AA^{-1}$ and $\sigma_{f_{\mathrm{b}, \lambda}} \sim 6 \times 10^{-16} \mathrm{erg} \mathrm{s}^{-1} \mathrm{~cm}^{-2} \AA^{-1}$, respectively. The latter value places a conservative upper limit on the detectable flux emitted by the blend and, ultimately, the lens. This flux density upper limit is equivalent to the magnitude's uppper limit of $\sim V \leq 17 \mathrm{mag}$. As presented in Table 2, the main sequence lens scenario predicts $V \sim 20 \mathrm{mag}$, which is much fainter than the previous limit. Therefore, the current spectroscopic data does not bring additional constraints on the nature of the lens. With a predicted blend flux ratio in the $V$-band, namely, $g_{v} \sim 2 \%$, we note that this analysis is consistent with the absence of blend light reported in the photometric results presented in $\mathrm{R} 21$.

\section{Conclusions}

In this work, we present spectroscopic follow-up studies of the microlensing event Gaia19bld. We collected several spectra on different instruments over the course of the event. For the first time, we performed a joint analysis of the absorption lines of the X-shooter and NRES high resolution, as well as a template-matching modeling. The spectra lines and template matching analysis converge to similar solutions. The source is a red giant $\left(T_{\text {eff }} \sim 4100 \mathrm{~K}, \log g \sim 1.5,[\mathrm{M} / \mathrm{H}] \sim 0.3\right.$ and $\theta_{*} \sim 24 \mu \mathrm{as}$ ), located at $\sim 8.4 \mathrm{kpc}$ from the Earth. We did not measure any significant blend light and, therefore, there is no detection of the lens in the spectroscopic data. Indeed, the combination of the measurement of $\theta_{*}$ and the parameters extracted from the light curve (R21) leads the a lens mass $\sim 1.1 M_{\odot}$ located at $D_{1} \sim 5.5 \mathrm{kpc}$. At this distance, a main sequence lens would be too faint to have been detected in spectroscopic (and photometric) data because it is much fainter $(V \sim 20 \mathrm{mag})$ than our detection limit ( $V \leq 17 \mathrm{mag}$ ).

This work demonstrates the potential of the spectroscopic follow-up of the microlensing event. It allows for the precise characterization of the source star stellar parameters and its angular radius, as well as the extinction along the line of sight. This is especially useful for events in the Galactic disk, where the distance to the source and the extinction are not well known. 
It is expected that the methods described in this work will be used routinely in the era of the new generation of all-sky surveys currently under development. In particular, the Legacy Survey of Space and Time (LSST Science Collaboration 2009) will detect thousands of events in the Galactic disk every year (Sajadian \& Poleski 2019). This will require similar spectroscopic monitoring in order to better characterize their properties and ultimately improve our understanding of faint objects throughout the entire Milky Way.

Acknowledgements. E.B. and R.S. gratefully acknowledge support from NASA grant 80NSSC19K0291. Y.T. acknowledges the support of DFG priority program SPP 1992 "Exploring the Diversity of Extrasolar Planets" (TS 356/3-1) This work is supported by Polish NCN grants: Daina No. 2017/27/L/ST9/03221, Preludium No. 2017/25/N/ST9/01253, Harmonia No. 2018/30/M/ST9/00311 and MNiSW grant DIR/WK/2018/12 as well as European Commission's Horizon2020 OPTICON grant No. 730890. This paper uses data collected with ESO/VLT/X-shooter instrument allocated via DDT programme No. 2103.D5046. We thank the ESO staff for their support. This work has made use of data from the European Space Agency (ESA) mission Gaia (https://www cosmos.esa.int/gaia), processed by the Gaia Data Processing and Analysis Consortium (DPAC, https://www. cosmos.esa.int/web/gaia/dpac/ consortium). Funding for the DPAC has been provided by national institutions, in particular the institutions participating in the Gaia Multilateral Agreement This research has made use of the VizieR catalogue access tool, CDS, Strasbourg, France (DOI: 10.26093/cds/vizier). The original description of the VizieR service was published in Ochsenbein et al. (2000). Softwares: Astropy (Astropy Collaboration 2018), emcee (Foreman-Mackey et al. 2013), Spyctres (https://github.com/ebachelet/Spyctres), pyLIMA (Bachelet et al. 2017), pysynphot (STScI Development Team 2013).

\section{References}

Adams, A. D., Boyajian, T. S., \& von Braun, K. 2018, MNRAS, 473, 3608

Alonso, A., Salaris, M., Arribas, S., Martínez-Roger, C., \& Asensio Ramos, A. 2000, A\&A, 355, 1060

Anders, F., Khalatyan, A., Chiappini, C., et al. 2019, A\&A, 628, A94

Astropy Collaboration (Price-Whelan, A. M., et al.) 2018, AJ, 156, 123

Bachelet, E., Shin, I. G., Han, C., et al. 2012, ApJ, 754, 73

Bachelet, E., Norbury, M., Bozza, V., \& Street, R. 2017, AJ, 154, 203

Bachelet, E., Beaulieu, J. P., Boisse, I., Santerne, A., \& Street, R. A. 2018, ApJ, 865,162

Bailer-Jones, C. A. L., Rybizki, J., Fouesneau, M., Mantelet, G., \& Andrae, R. 2018, AJ, 156, 58

Beaulieu, J. P., Bennett, D. P., Batista, V., et al. 2016, ApJ, 824, 83

Bensby, T., Yee, J. C., Feltzing, S., et al. 2013, A\&A, 549, A147

Berger, T. A., Huber, D., Gaidos, E., \& van Saders, J. L. 2018, ApJ, 866, 99

Blanco-Cuaresma, S. 2019, MNRAS, 486, 2075

Blanco-Cuaresma, S., Soubiran, C., Heiter, U., \& Jofré, P. 2014, A\&A, 569, A111

Blanton, M. R., \& Roweis, S. 2007, AJ, 133, 734

Boisse, I., Santerne, A., Beaulieu, J. P., et al. 2015, A\&A, 582, L11

Boyajian, T. S., van Belle, G., \& von Braun, K. 2014, AJ, 147, 47

Brahm, R., Jordán, A., \& Espinoza, N. 2017, PASP, 129, 034002

Bressan, A., Marigo, P., Girardi, L., et al. 2012, MNRAS, 427, 127

Brown, T. M., Baliber, N., Bianco, F. B., et al. 2013, PASP, 125, 1031

Cardelli, J. A., Clayton, G. C., \& Mathis, J. S. 1989, ApJ, 345, 245

Carnall, A. C. 2017, ArXiv e-prints [arXiv:1705. 05165]

Cassan, A., \& Ranc, C. 2016, MNRAS, 458, 2074
Cassan, A., Ranc, C., Absil, O., et al. 2021, Nat. Astron., https://doi .org/ 10. 1038/s41550-021-01514-w

Cutri, R. M., Skrutskie, M. F., van Dyk, S., et al. 2003, VizieR Online Data Catalog: II/246

DENIS Consortium 2005, VizieR Online Data Catalog: II/263

Dominik, M., \& Sahu, K. C. 2000, ApJ, 534, 213

Dong, S., Mérand, A., Delplancke-Ströbele, F., et al. 2019, ApJ, 871, 70

Foreman-Mackey, D., Hogg, D. W., Lang, D., \& Goodman, J. 2013, PASP, 125 306

Fukui, A., Suzuki, D., Koshimoto, N., et al. 2019, AJ, 158, 206

Gaia Collaboration (Prusti, T., et al.) 2016, A\&A, 595, A1

Gaia Collaboration (Brown, A. G. A., et al.) 2021, A\&A, 649, A1

Girard, T. M., van Altena, W. F., Zacharias, N., et al. 2011, VizieR Online Data Catalog: I/320

Gould, A. 2004, ApJ, 606, 319

Grevesse, N., Asplund, M., \& Sauval, A. J. 2007, Space Sci. Rev., 130, 105

Gustafsson, B., Edvardsson, B., Eriksson, K., et al. 2008, A\&A, 486, 951

Han, C. 2008, ApJ, 681, 806

Hodgkin, S. T., Wyrzykowski, L., Blagorodnova, N., \& Koposov, S. 2013, Philos. Trans. R. Soc. London Ser. A, 371, 20120239

Horne, K. 1986, PASP, 98, 609

Jennings, J., \& Levesque, E. M. 2016, ApJ, 821, 131

Kervella, P., \& Fouqué, P. 2008, A\&A, 491, 855

Kurucz, R. L. 1993, SYNTHE Spectrum Synthesis Programs and Line Data (Cambridge, Mass.: Smithsonian Astrophysical Observatory)

LSST Science Collaboration (Abell, P. A., et al.) 2009, ArXiv e-prints [arXiv:0912.0201]

Lu, J. R., Sinukoff, E., Ofek, E. O., Udalski, A., \& Kozlowski, S. 2016, ApJ, 830, 41

Luri, X., Brown, A. G. A., Sarro, L. M., et al. 2018, A\&A, 616, A9

Moehler, S., Modigliani, A., Freudling, W., et al. 2014, A\&A, 568, A9

Mróz, P., Udalski, A., Szymański, M. K., et al. 2020, ApJS, 249, 16

Ochsenbein, F., Bauer, P., \& Marcout, J. 2000, A\&AS, 143, 23

Penny, M. T., Henderson, C. B., \& Clanton, C. 2016, ApJ, 830, 150

Rybicki, K. A., Wyrzykowski, Ł., Klencki, J., et al. 2018, MNRAS, 476, 2013

Rybicki, K., Wyrzykowski, L., Zielinski, P., et al. 2019, ATel, 12948, 1

Rybicki, K., Wyrzykowski, Ł., Bachelet, E., et al. 2022, A\&A, 657, A18

Sajadian, S., \& Poleski, R. 2019, ApJ, 871, 205

Santerne, A., Beaulieu, J. P., Rojas Ayala, B., et al. 2016, A\&A, 595, L11

Schlafly, E. F., \& Finkbeiner, D. P. 2011, ApJ, 737, 103

Schlegel, D. J., Finkbeiner, D. P., \& Davis, M. 1998, ApJ, 500, 525

Siverd, R. J., Brown, T. M., Barnes, S., et al. 2018, SPIE Conf. Ser., 10702 $107026 \mathrm{C}$

Skowron, J., Udalski, A., Gould, A., et al. 2011, ApJ, 738, 87

Smith, M. C., Mao, S., \& Paczyński, B. 2003, MNRAS, 339, 925

Street, R. A., Udalski, A., Calchi Novati, S., et al. 2016, ApJ, 819, 93

STScI Development Team. 2013, Astrophysics Source Code Library [record ascl: 1303.023$]$

Tsapras, Y. 2018, Geosciences, 8, 365

Udalski, A., Yee, J. C., Gould, A., et al. 2015, ApJ, 799, 237

Vernet, J., Dekker, H., D’Odorico, S., et al. 2011, A\&A, 536, A105

Weiler, M. 2018, A\&A, 617, A138

Witt, H. J., \& Mao, S. 1994, ApJ, 430, 505

Wyrzykowski, Ł., \& Hodgkin, S. 2012, IAU Symp., 285, 425

Wyrzykowski, Ł., Mróz, P., Rybicki, K. A., et al. 2020, A\&A, 633, A98

Yee, J. C., Johnson, J. A., Skowron, J., et al. 2016, ApJ, 821, 121

Yoo, J., DePoy, D. L., Gal-Yam, A., et al. 2004, ApJ, 603, 139

Zacharias, N., Monet, D. G., Levine, S. E., et al. 2005, VizieR Online Data Catalog: I/297 